\title{
Radial Gradients of Abundances
}

\author{
Mercedes Mollá and Angeles I.Díaz \\ Dept. Física Teórica, Universidad Autónoma de Madrid, 28049 Cantoblanco, Spain \\ F. Ferrini \\ INTAS, 58 Avenue des Arts, 1000 Bruxelles, Belgium
}

\begin{abstract}
We have computed a set of multiphase chemical evolution models in which the radial mass distribution of each theoretical galaxy is calculated using the universal rotation curve from Persic, Salucci \& Steel (1996). We obtain the chemical evolution for galaxies of different masses and morphological types by changing the efficiencies to form molecular clouds and stars according with these types.

We obtain the radial distribtions of diffuse and molecular gas densities, the star formation rate and abundances for 15 elements for each galaxy.
\end{abstract}

Keywords: galaxies: evolution, abundances:oxygen

\section{The generalization of the multiphase evolution model}

The multiphase chemical evolution model assumes a spherical protogalaxy with a gas mass which collapses to fall onto the equatorial plane forming the disk as a secondary structure.

The radial distribution of mass of each theoretical galaxy is calculated through the Universal Rotation Curve from Persic et al. (1996). The infall rate of gas from a halo region to the disk is inversely proportional to a collapse time scale which depends on the total mass being shorter for the more massive galaxies.

The stars in the disks form in two steps: molecular clouds form from the diffuse gas by a Schmidt law, and then cloud-cloud collisions produce stars by a spontaneous process. The efficiencies $\epsilon_{\mu}$ and $\epsilon_{h}$ to form clouds or stars by these two processes are assumed to depend on morphological or Hubble type T, such as it was found in our previous investigations. For futher details, see Mollá et al. (2001).

\section{Results: The behaviour of oxygen abundances radial gradients}

Under these assumptions, we obtain the radial distributions of diffuse and molecular gas densities, the star formation rate and abundances for 15 elements for each galaxy. 

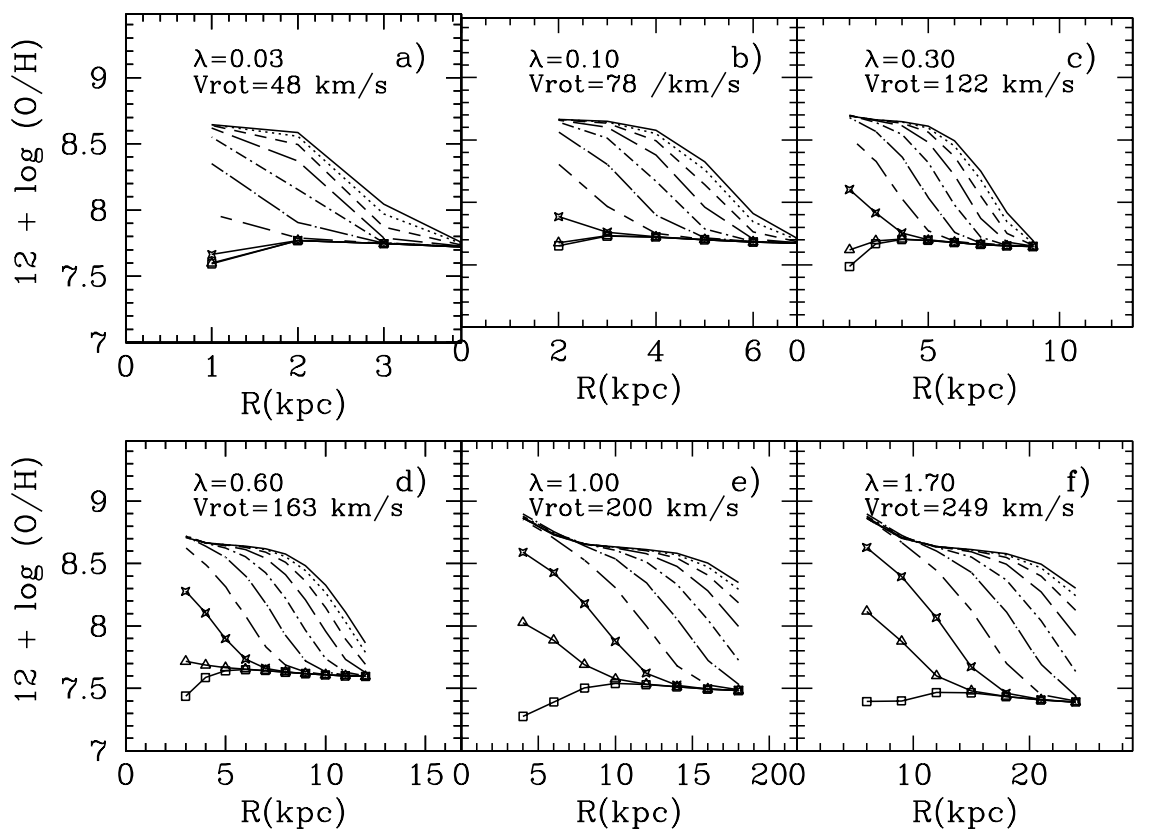

Figure 1. The oxygen abundance radial distributions for six different values of galaxy mass. En each panel 10 different models are represented corresponding to 10 Hubble $\mathrm{T}$, with the most evolved ones at the top and the less ones at the bottom.

The most important outcome concerns the oxygen abundance: the radial gradient only appears for the intermediate types $(7<\mathrm{T}<4)$ at all galaxy masses, being larger for the less massive galaxies. However, the latest ones $(\mathrm{T} \geq 8)$ show a flat gradient with abundances $12+$ $\log (O / H) \sim 7.5-8$. This solves the apparent inconsistency of the largest gradients appearing in late type spirals while some irregulars shows no gradient at all.

Results, which can see in Fig. 1, are in close agreement with observations for HSB, LSB and dwarf galaxies. Variations of the radial gradients of abundances with the morphological type and with the total mass of the galaxy follow the observed correlations.

\section{References}

Mollá M., Díaz, A.I. \& Ferrini, F. 2001, in preparation

Persic, M., Salucci, P. \& Steel, F. 1996, MNRAS, 281, 27 